\title{
Dynamics of electron-capture-to-continuum (ECC) formation in slow ion-atom collisions
}

\author{
F Afaneh ${ }^{1}$, L Ph H Schmidt ${ }^{2}$, M Schöffler ${ }^{2}$, K E Stiebing ${ }^{2}$, J Al-Jundi ${ }^{1}$, \\ H Schmidt-Böcking ${ }^{2}$ and R Dörner ${ }^{2}$ \\ ${ }^{1}$ Physics Department, The Hashemite University, PO Box 150459, Zarqa 13115, Jordan \\ ${ }^{2}$ Institut für Kernphysik, Max-von-Laue-Str. 1, 60438 Frankfurt am Main, Germany \\ E-mail: afaneh@hu.edu.jo
}

Received 11 February 2007, in final form 22 March 2007

Published 30 April 2007

Online at stacks.iop.org/JPhysB/40/1745

\begin{abstract}
The zero-degree ejected-electron spectrum for protons incident on $\mathrm{He}$ at $25 \mathrm{keV}$ is examined experimentally using the COLTRIMS technique. The momentum distribution of the emitted electrons for the transfer ionization (TI) reaction channel is measured in coincidence with the momentum vectors of the recoil ion and the scattered projectile. The momentum distribution of the electrons emitted around zero degree in the forward direction for the TI reaction channel shows two prominent structures: the electron-capture-to-the-continuum (ECC) peak and the saddle-point peak. From the measured fully differential electron emission cross sections with respect to the scattering plane we can deduce that the main ECC formation mechanism is electron promotion via quasimolecular orbitals.
\end{abstract}

\section{Introduction}

To fully understand the ionization processes in ion-atom collisions from both an experimental and theoretical perspective, most of the experimental and theoretical works done over the past 2 decades have been focused on fully differential cross sections. There are several characteristic structures in the electron spectra. At least two distinct features in the electron emission spectra in ion-atom collisions have been identified: the electron capture to the continuum (ECC) peak found in the forward direction of the differential cross sections where the velocity of the ejected electron $\left(V_{\mathrm{e}}\right)$ matches that of the projectile $\left(V_{\mathrm{p}}\right)$ and the binary-encounter $(\mathrm{BE})$ ridge. The ECC electrons form a peak structure only if the differential cross sections are shown for a narrow angular range around zero degree. This cusp-shaped peak was first observed by Crooks and Rudd [1]. The other structure, which is the BE ridge, can be identified as a circular ridge centred around the incoming projectile velocity vector. At zero degree this yields an electron velocity which is approximately twice that of the projectile. A third feature in the 
forward direction, a peak at the saddle point, is under debate. It has been suggested that the saddle-point emission mechanism arises from the possibility that the electrons removed from the target could escape capture if they are stranded on the saddle in the two-centre potential between the residual target and receding projectile [2-4].

The study of the production mechanisms of the electron capture to the continuum (ECC) peak has been one of the most popular objectives both experimentally [5-14] and theoretically [15-21] since its discovery [1]. Most of the experiments have been performed using electron spectroscopy [1,5-8]. Over the past few years, the experimental techniques have advanced considerably with the use of imaging techniques such as the recoil-ion momentum spectroscopy. They allow measuring the full momentum space distribution of the low-energy ejected electrons for fixed values of the vector impact parameter [9-13]. Using the recoil-ion momentum spectroscopy, Jagutzki and co-workers [9] were able for the first time to study the impact parameter dependence of the ECC structure for the collision systems $0.53 \mathrm{MeV} / \mathrm{u}$ $\mathrm{Cu}^{5+}$ and $\mathrm{Cu}^{15+}$ on $\mathrm{He}$. Recently in their study of the single ionization process for the $\mathrm{d}^{+}-\mathrm{He}$ collision system at high impact energies employing the COLTRIMS technique, Weber and co-workers have found an abrupt rise in the longitudinal $\mathrm{He}^{+}$recoil-ion momentum distribution that is related to the ECC processes [12].

On the theoretical side, earlier models showed that the ECC peak can be viewed as an extrapolation across the ionization limit of capture into highly excited bound states [15]. A different detailed picture of the ECC peak based on the classical trajectory Monte Carlo (CTMC) calculations was given by Reinhold and Olson [16]. They showed that the ECC peak can be considered as a classical phenomenon and is formed at large distances. They found that at these large distances, electron-projectile interactions play an essential role in the formation of the cusp. It is generally assumed that the ECC peak appearing in the spectrum of the forward emitted electrons in ion-atom collisions is to be a divergence [20]. Recently Illescas et al [21] claimed, however, that the ECC peak is not a divergence smoothed by the experiment, and is slightly shifted from the impact-velocity value. They have explained the ECC emission mechanism classically by the following mechanism: in the early stage of the process, ECC electrons are temporarily captured by the projectile in Rydberg states through a Coulomb focusing process. In the outgoing phase of the collision the Coulomb field of the receding target ion perturbs the motion of the electrons, leading to ionization. These results have been confirmed in the experimental work of Shah et al [14]. They measured the ECC cusp for collisions of 10 and $20 \mathrm{keV}$ protons with $\mathrm{H}_{2}$ and $\mathrm{He}$, and found that the peak was indeed shifted to a velocity below its expected position.

Since the saddle-point mechanism has been extensively investigated in our previous studies [22-25], in this study we will focus attention on the role of the ECC in slow ionatom collisions. In the present study of the ECC emission, we investigate p-He collisions at $25 \mathrm{keV}$. The momentum distributions of emitted electrons were measured in coincidence with the momentum vectors of the recoil ion and the scattered projectile using the cold target recoil ion momentum spectroscopy (COLTRIMS) technique.

\section{Experiment}

The experiment was performed at the ECRIS of the Institute for Nuclear physics of the Frankfurt University using cold target recoil ion momentum spectroscopy (COLTRIMS) $[26,27]$. A schematic picture of the experimental apparatus is shown in figure 1 . An ion beam intersects a supersonically cooled He gas jet at right angles. The gas jet consists of two stages. The He gas at 17 bar expands through a $30 \mu \mathrm{m}$ nozzle into the first expansion chamber at a vacuum of $10^{-1}$ mbar. The large pressure difference between the reservoir and 


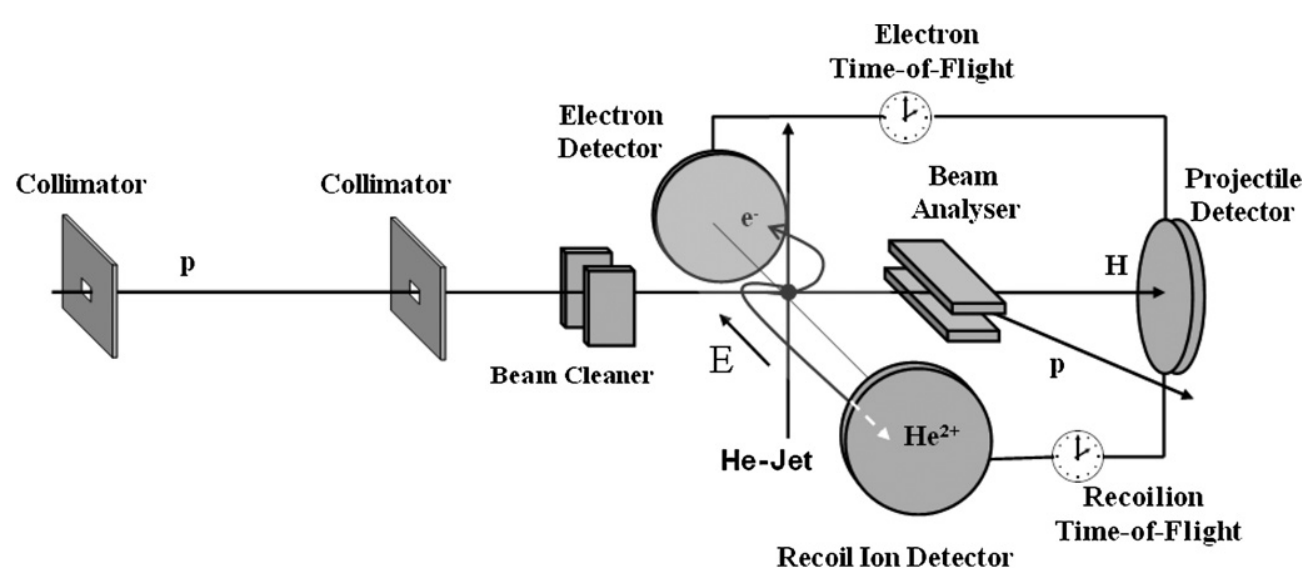

Figure 1. Schematic experimental setup.

the surrounding region leads to adiabatic expansion of the He gas resulting in cooling of the beam in the direction of the pressure gradient. $3 \mathrm{~mm}$ above the nozzle the central part of the gas jet enters into the second expansion chamber which is at $5 \times 10^{-5}$ mbar through a skimmer of $0.3 \mathrm{~mm}$ diameter. A second skimmer of $0.4 \mathrm{~mm}$ diameter is placed at a distance of $30 \mathrm{~mm}$ from the nozzle and separates the second chamber from the collision chamber. The gas jet leaves the collision chamber through a $10 \mathrm{~mm}$ hole into a separately pumped jet dump. A gas jet of $1.1 \mathrm{~mm}$ diameter at the reaction region was obtained. The target density was estimated from the gas flow into the jet dump to be $2 \times 10^{11} \mathrm{~cm}^{-3}$ while the residual gas density at the collision chamber is $2 \times 10^{9} \mathrm{~cm}^{-3}$. The gas jet is crossed with the proton beam extracted from the ECR ion source. The $\mathrm{H}^{+}$beam is collimated by two sets of adjustable slits to a beam-spot size of $1 \mathrm{~mm}^{2}$ at the interaction point. $20 \mathrm{~cm}$ upstream of the intersection point a set of electrostatic deflectors allows one to clean the beam from charge state impurities. $20 \mathrm{~cm}$ downstream of the target a second set of deflectors separates the final projectile charge states. The projectiles which captured an electron are then detected by a two-dimensional position-sensitive channel plate detector.

At the target region a homogeneous electric field of $13 \mathrm{~V} \mathrm{~mm}^{-1}$ is applied perpendicular to the plane defined by the projectile beam (z-axis) and gas jet ( $y$-axis). This field projects the ionized target ions and the electrons in opposite directions onto two micro channel-plate detectors with delay line anodes aligned in the $y z$-plane [28]. The recoil ions created at the intersection point were accelerated by passing a $35 \mathrm{~mm}$ extraction field region. The ions were then entered a $128 \mathrm{~mm}$ drift tube. Drift and acceleration regions are separated by a woven mesh. A combination of a lens and a drift region has been used to optimize the momentum resolution of the recoil ion spectrometer and to eliminate the degrading influence of the final size of the collision range (three-dimensional position and time focusing conditions) [29, 30]. At the end of the drift tube the ions passed a stack of three grids and were post accelerated with $2000 \mathrm{~V}$ onto a position-sensitive channel-plate detector. On the opposite side the emitted electrons were accelerated by a $15 \mathrm{~mm}$ long extraction field. After acceleration the electrons passed a drift tube of $40 \mathrm{~mm}$ length and were detected by another position-sensitive channelplate detector shifted by $12 \mathrm{~mm}$ forward in the beam direction with respect to the reaction zone. The time of flights and positions of all collision products were simultaneously recorded with a $0.5 \mathrm{~ns}$ resolution by a 15-channel multi-hit time-to-digital converter (LeCroy TDC 3377). The TDC was operated in a common stop mode, i.e. where the signals were measured 


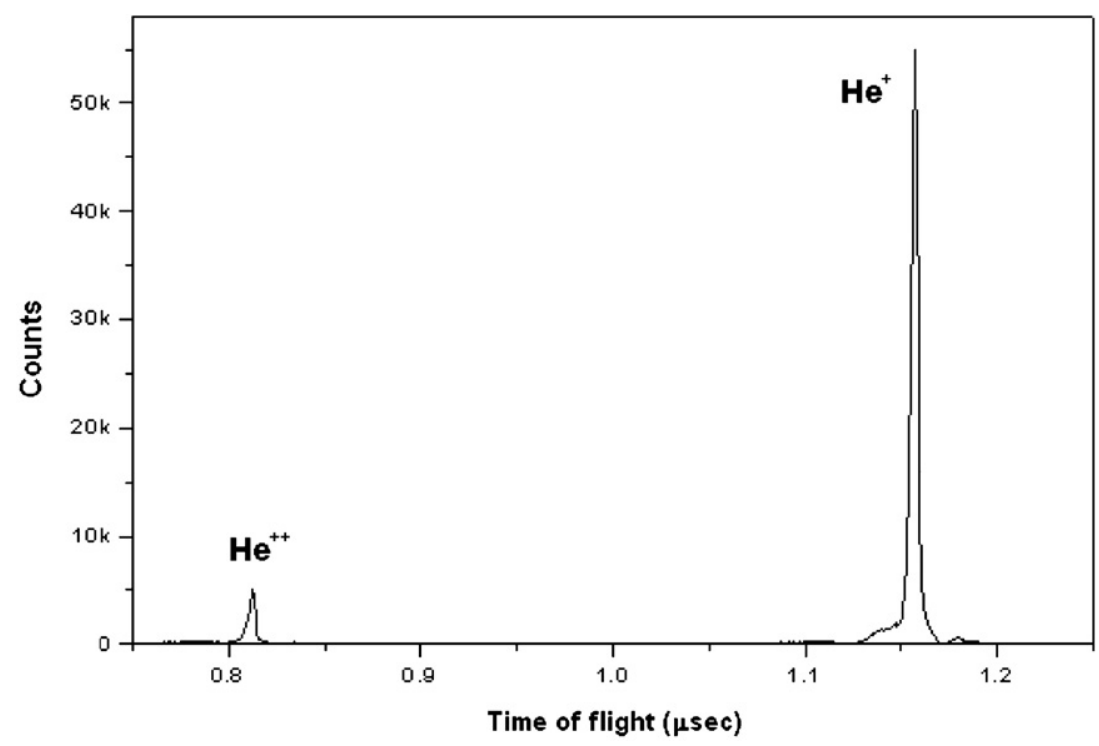

Figure 2. Time-of-flight distribution of He recoil ions measured in coincidence with the emitted electron (single ionization reaction channel: right peak) and scattered projectiles (transfer ionization reaction channel: left peak). The width of the peaks reflects the momentum distribution of the recoil ions in the direction of the electric field.

to a delayed common stop signal made by the coincidence between electron and recoil ion timing signals. Finally, the momentum vector of the recoil ion and the emitted electron were calculated from the times of flight and the positions on the detectors.

\section{Results and discussion}

Figure 2 shows the time-of-flight distribution of the recoil ions measured with respect to the electron timing signal. The distribution shows two peaks each of them represents one reaction channel. While the right peak in figure 2 represents the $\mathrm{He}^{+}$recoil ions produced in the single ionization reaction,

$$
\mathrm{H}^{+}+\mathrm{He} \rightarrow \mathrm{H}^{+}+\mathrm{He}^{+}+\mathrm{e}^{-} .
$$

The left peak in figure 2 belongs to the $\mathrm{He}^{++}$recoil ions produced in the transfer ionization reaction. The later reaction channel was distinguished from the double ionization by the coincident detection of the neutral scattered projectiles

$$
\mathrm{H}^{+}+\mathrm{He} \rightarrow \mathrm{H}^{0}+\mathrm{He}^{++}+\mathrm{e}^{-} \text {. }
$$

From the time of flight one obtains the charge state and the momentum component of the recoil ion in the field direction ( $X$-axis). The two momentum components perpendicular to the field are calculated from the position on the recoil ion detector. The momentum vector of the recoil ions has been calculated for the transfer ionization (TI) reaction channel. Figure 3 shows the transverse momentum distribution of the $\mathrm{He}^{++}$recoil ions resulting from the TI reaction channel. The resolution of the $\mathrm{He}^{++}$recoil transverse momentum was limited to 0.4 au (FWHM) by the broader momentum distribution of the gas target in the jet direction. 


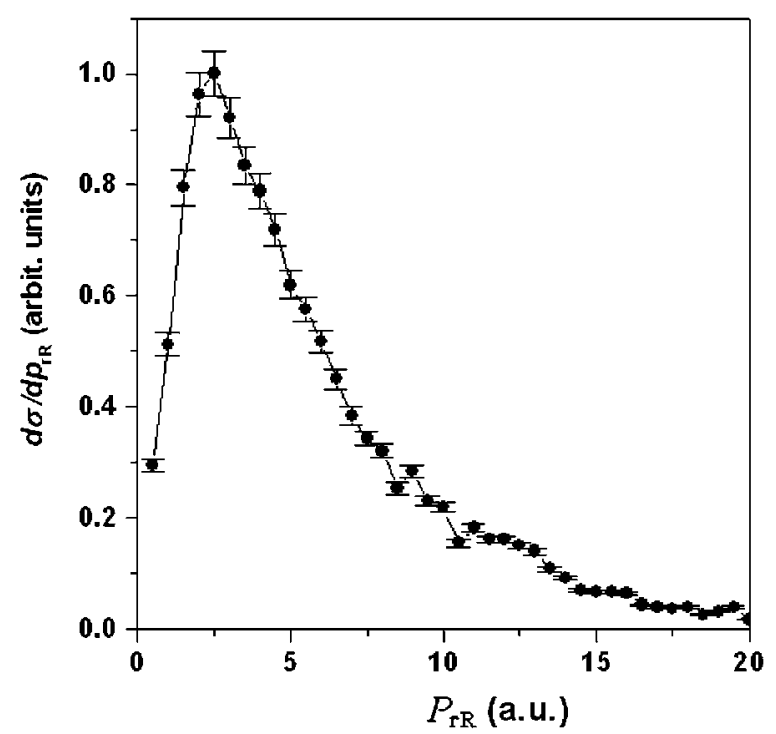

Figure 3. Single differential cross sections $\mathrm{d} \sigma / \mathrm{d} p_{\mathrm{rR}}$ of the TI reaction channel at projectile velocity $V_{\mathrm{p}}=1 \mathrm{au}$ as a function of the transverse momentum of the $\mathrm{He}^{++}$recoil ions. The distribution peaks at $P_{\mathrm{rR}} \approx 2.5 \mathrm{au}$ which correspond to an impact parameter of about $1 \mathrm{au}, P_{\mathrm{rR}} \approx 10$ and 20 au correspond to impact parameters in the range of 0.2 and 0.1 au, respectively.

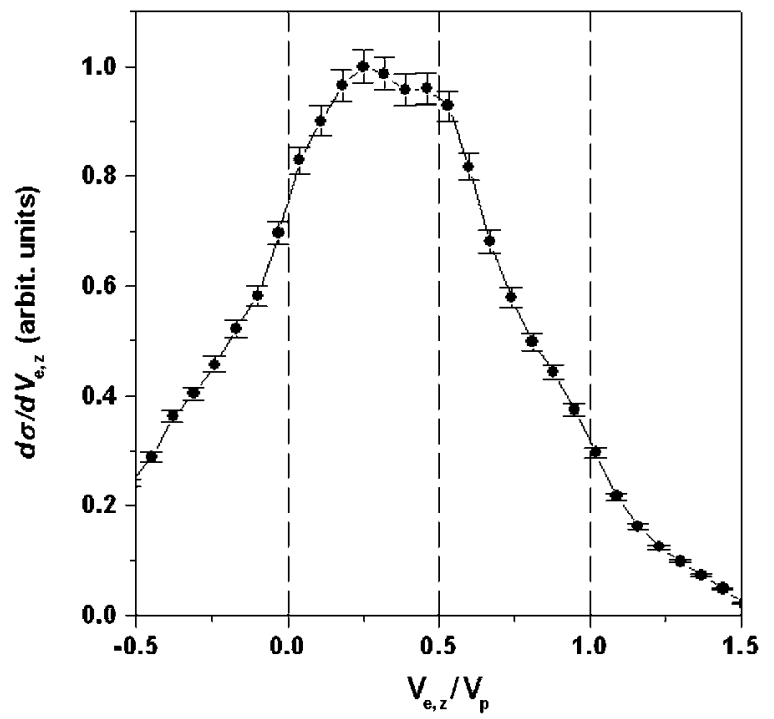

Figure 4. The single differential longitudinal momentum distribution of the emitted electrons scaled by the projectile velocity is shown for the TI reaction channel integrated over all electron emission angles. The dashed lines mark the characteristic beam velocities.

Figure 4 displays the longitudinal momentum distribution of the emitted electron for the TI reaction channel integrated over all electron emission angles and averaged over all recoil momenta, i.e. it reflects a single differential longitudinal electron momentum spectrum for the TI channel. The resolution achieved in electron velocity distribution is limited by the width of the projectile beam $(1 \mathrm{~mm})$ in the $Y$-direction and the width of the target gas jet $(1.1 \mathrm{~mm})$ in 


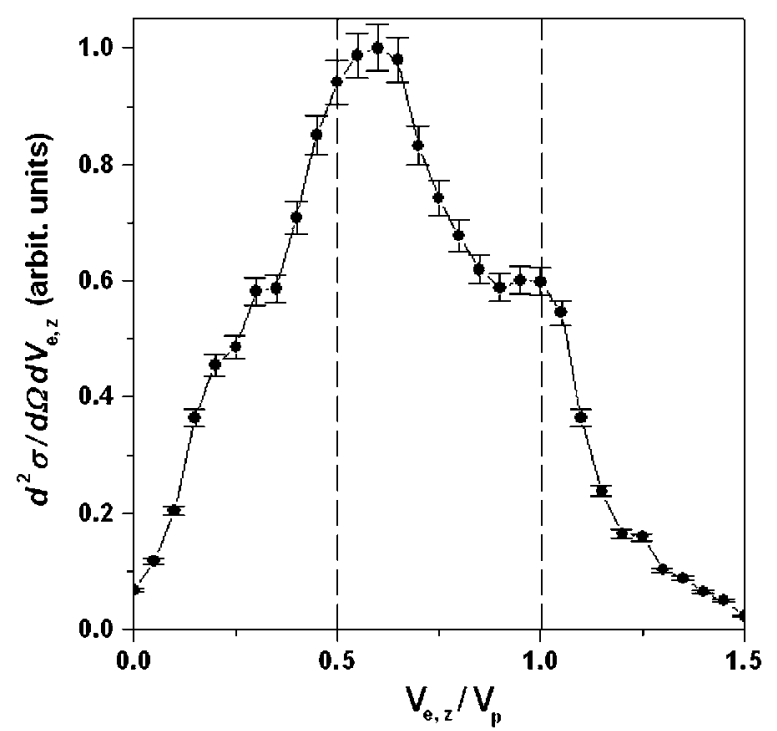

Figure 5. The longitudinal momentum distribution of the electrons emitted near zero degree $\left(\Delta= \pm 5^{\circ}\right)$ for the TI reaction channel, scaled by the projectile velocity.

the $Z$-direction. The electron velocity resolution in the $Z$-direction is approximately 0.12 au (FWHM). In this single differential longitudinal momentum distribution of the emitted electron presented here we deduce that the electrons are preferentially emitted in the forward direction with a broad maximum in the vicinity of the saddle-point velocity ( $0<V_{\mathrm{e}}<V_{\mathrm{p}}$ ) (see also [31]). These electrons are known as saddle-point electrons. This overall spectral feature was predicted by Olson et al [3]. The distribution shown in figure 4 is completely different from the electron-momentum distributions for the TI channel of the same collision system at higher velocities [32-34]. At higher velocities the main TI channel is electron capture via the non-s ${ }^{2}$ contributions of the He ground state and thus the second electron is emitted via shake-off processes. The final state momentum pattern of the shake-off electron is thus only determined by the momentum correlation in the ground state wavefunction.

To study the ECC emission, the longitudinal momentum distribution of the emitted electron was generated at electron emission angles in a narrow cone around zero degree $(\Delta=$ $\pm 5^{\circ}$ ). The distribution is employed to examine the ECC formation mechanisms that played a role in the transfer ionization process.

The single differential longitudinal momentum distribution of the electrons emitted near zero degree $\left(\Delta= \pm 5^{\circ}\right)$ for the TI reaction channel is presented in figure 5 . Both ionization features, i.e. ECC and saddle-point emission can be observed in the longitudinal momentum distribution. The figure implies also that the saddle-point mechanism is the main emission process in the TI reaction channel.

As stated above we will focus on the study of the dynamics of the electron-capture-tocontinuum (ECC) emission. To explore this dynamics one has to measure the fully differential electron emission distribution with respect to the nuclear scattering plane to reveal all details of the electron emission along the beam axis. In figure 6 the fully differential electron emission for the TI reaction channel is shown for two different ranges of recoil transverse momenta.

Figure 6 clearly shows that the electron emission is asymmetric with respect to the beam axis and is favoured towards the direction of the recoiling ion. The banana shape distribution 

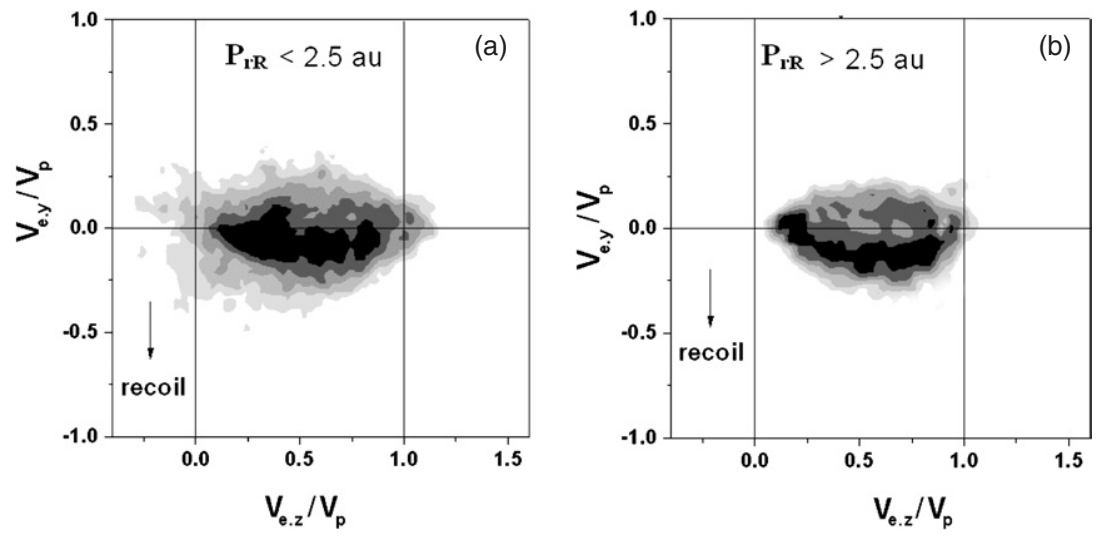

Figure 6. Momentum distribution of the emitted electron in the scattering plane defined by the beam direction ( $z$-axis) and the recoil ion ( $-y$-axis; i.e., the scattered projectile is $+y$-axis) (a) for small recoil transverse momentum transfer and (b) for large recoil transverse momentum transfer.
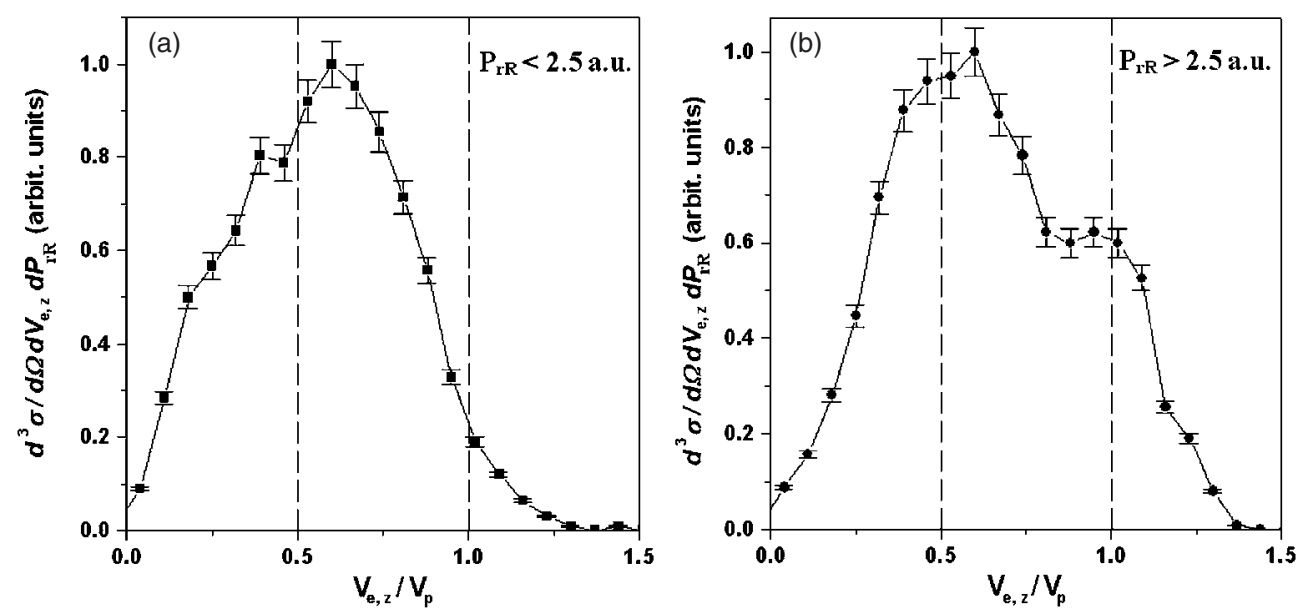

Figure 7. The longitudinal momentum distribution of the electrons emitted near zero degree $\left(\Delta= \pm 5^{\circ}\right)$ for the TI reaction channel (a) for small recoil transverse momentum transfer $\left(P_{\mathrm{rR}}<\right.$ $2.5 \mathrm{au})$ and (b) for large recoil transverse momentum transfer $\left(P_{\mathrm{rR}}>2.5 \mathrm{au}\right)$.

in the scattering plane is not in agreement with the scaling of saddle-point electron emission but strongly supports electron promotion via quasimolecular orbitals $[35,36]$. Thus the data strongly support that the dynamics creating ECC electrons is quasimolecular promotion. Different to the observations in [32-34] He ground state correlation does not have any influence on the ECC formation.

Finally if singly differential electron spectra with respect to the longitudinal momenta for different ranges of recoil transverse momenta are created along a narrow cone around zero degree even the ECC peak becomes visible. These results are presented in figures 7(a) and (b). In these figures the recoil transverse momentum transfer increases from (a) to (b) in figure 7 , therefore the impact parameter decreases in the same direction. These figures indicate a strong impact parameter dependence of the ECC structure. At small recoil transverse momentum transfer $\left(P_{t}<2.5 \mathrm{au}\right)$ (large impact parameters $\left.(b>1 \mathrm{au})\right)$, the electron singly differential 
longitudinal momentum distribution exhibits the saddle-point peak. No ECC peak can be observed. In contrast, at large recoil transverse momentum transfer $\left(P_{t}>2.5 \mathrm{au}\right.$ ) (small impact parameters $(b<1 \mathrm{au})$ ), both saddle-point and ECC peaks are present in the longitudinal momentum distribution. It is also interesting to note that the ECC does not exactly peak at the projectile velocity as predicted. It is slightly shifted to lower momenta. This is consistent with the findings of Shah et al [14]. The ECC peak shift has been attributed to post collisional effects that follow from the pull of the target on the ionized electron after it becomes free, and is therefore clear outcome of the so-called two-centre effects [14, 21].

\section{Conclusions}

In conclusion, we used the well-known COLTRIMS imaging technique to measure the momentum distribution of electrons emitted around zero degree for the collision system $25 \mathrm{keV}$ protons on helium. The momentum distribution of the emitted electrons was measured for the transfer ionization (TI) reaction channel in coincidence with both the recoil ion momentum vector and the scattered projectiles. Two different peaks are observed in the momentum distributions of the electrons emitted around zero degree. These peaks result from the electron capture to the continuum (ECC) and the saddle-point mechanism. The impact parameter dependence of both emission mechanisms was examined. It is found that the ECC peak results from a narrow range of impact parameters.

\section{Acknowledgments}

FA gratefully acknowledges support from the Hashemite University, the DAAD and the DFG. We thank RoentDek Handels GmbH for the preparation of the position sensitive detectors and other technical equipment. This work is supported by DFG, the Hashemite University, DAAD and RoentDek GmbH.

\section{References}

[1] Crooks G B and Rudd M E 1970 Phys. Rev. Lett. 251599

[2] Olson R E 1983 Phys. Rev. A 271871

[3] Olson R E 1986 Phys. Rev. A 334397

[4] Pieksma M, Ovchinnikov S, van Eck J, Westerveld W and Niehaus A 1994 Phys. Rev. Lett. 7346

[5] Lucas M W and Harrison K G 1972 J. Phys. B: At. Mol. Phys. 5 L20

[6] Meckbach W, Nemirovsky I B and Garibotti C R 1981 Phys. Rev. A 241793

[7] Bernardi G, Suarez S, Fainstein P, Garibotti C, Meckbach W and Focke P 1989 Phys. Rev. A 406863

[8] Sarkadi L, Brinkmann U, Bader A, Hippler R, Tokesi K and Gulyas L 1998 Phys. Rev. A 58296

[9] Jagutzki O et al 1991 J. Phys. B: At. Mol. Opt. Phys. 242579

[10] Hagmann S et al 1992 J. Phys. B: At. Mol. Opt. Phys. 25 L287

[11] McGrath C et al 2000 J. Phys. B: At. Mol. Opt. Phys. 333693

[12] Weber Thet al 2001 Phys. Rev. Lett. 86224

[13] An L, Khayyat Kh and Schulz M 2001 Phys. Rev. A $63030703 R$

[14] Shah M B et al 2003 Phys. Rev. A 67010704

[15] Macek J et al 1981 Phys. Rev. Lett. 461571

[16] Reinhold C O and Olson R E 1989 Phys. Rev. A 393861

[17] Brauner M and Briggs J S 1991 J. Phys. B: At. Mol. Opt. Phys. 242227

[18] Macri P A and Barrachina R O 1998 J. Phys. B: At. Mol. Opt. Phys. 311303

[19] Stolterfoht N, DuBois R D and Rivarola R D 1997 Electron Emission in Heavy Ion-Atom Collisions (Berlin: Springer)

[20] Rodriguez V D, Wang Y D and Lin C D 1995 Phys. Rev. A 52 R9

[21] Illescas C, Pons B and Riera A 2002 Phys. Rev. A 65030703 
[22] Dörner R et al 1996 Phys. Rev. Lett. 774520

[23] Afaneh F, Dörner R, Schmidt L and Schmidt-Böcking H 2005 Nucl. Instrum. Methods Phys. Res. B 234431

[24] Afaneh F et al 2002 J. Phys. B: At. Mol. Opt. Phys. 35 L229

[25] Schmidt L et al 2004 Phys. Scr. T 110379

[26] Dörner R, Mergel V, Jagutzki O, Spielberger L, Ullrich J, Moshammer R and Schmidt-Böcking H 2000 Phys. Rep. 33096

[27] Ullrich J, Moshammer R, Dorn A, Dörner R, Schmidt L Ph and Schmidt-Böcking H 2003 Rep. Prog. Phys. 661463

[28] Jagutzki O et al 2002 Nucl. Instrum. Methods Phys. Res. A 477244

[29] Mergel V 1994 Diploma Thesis Johann-Wolfgang Universität Frankfurt

[30] Dörner R et al 1997 Nucl. Instrum. Methods. Phys. Res. B 124225

[31] Kravis S D et al 1996 Phys. Rev. A 541394

[32] Mergel V, Dörner R, Khayyat Kh, Achler M, Weber T, Schmidt-Böcking H and Lüdde H J 2001 Phys. Rev. Lett. 862257

[33] Mergel V et al 1997 Phys. Rev. Lett. 79387

[34] Schöffler M et al 2005 J. Phys. B: At. Mol. Opt. Phys. 38 L123

[35] Macek J H and Ovchinnikov S Y 1994 Phys. Rev. A 50468

[36] Ovchinnikov S Y and Macek J H 1995 Phys. Rev. Lett. 752474 